\title{
An Overheard-Based Relay-Assisted MAC Protocol Using Symbol Level Network Coding in Vehicular Networks
}

\author{
Yuh-Shyan Chen \\ Department of CSIE \\ National Taipei University \\ Taipei 151, Taiwan, R. O. C. \\ Email:yschen@mail.ntpu.edu.tw
}

\author{
Chih-Shun Hsu \\ Department of Info. Management \\ Shih Hsin University \\ Taipei, 116 Taiwan, R. O. C. \\ Email: cshsu@cc.shu.edu.tw
}

\author{
Jhao-Yen Wei \\ Institute of Info. Management \\ National Taipei University \\ Taipei 151, Taiwan, R. O. C. \\ Email: goodfifagun@gmail.com
}

\begin{abstract}
Many network coding schemes have been proposed so as to enhance the communication efficiency and security of wireless networks. To further enhance the communication efficiency, an overheard-based relay-assisted MAC protocols for vehicular networks is proposed in this paper. Since the symbol level network coding (SLNC) scheme has lower error probability than the packet level network coding (PLNC) scheme, SLNC is adopted in the proposed protocol so as to improve the transmission efficiency. An overheard relay vehicle is chosen to help the avoidance of different data multiplying with the same coefficient. When the original data cannot be decoded due to the lost of some symbols, the symbol acquisition process is initiated. Simulation results have shown that the proposed protocol performs better than the traditional network coding protocol. It can reduce packet delivery delay, improve throughput and the successful probability of transmissions.
\end{abstract}

Keywords-network coding; symbol level network coding; packet level network coding

\section{INTRODUCTION}

Network coding [1] can be used to reduce redundant transmissions in wireless networks. It changes the original communication network by a method, which calls "code" to encode the data packet, and then sends out from the source to the destination. When the destination node receives enough encoded packets, it can decode the original packets by linear elimination. Network coding greatly decreases the number of transmissions and increases throughput of the networks, and can also enhance security because the coded packets are totally different from original packets.

In vehicular networks, the network topology is changing quickly because of vehicles' high mobility, which may cause higher packet lost rate. To reduce the packet lost rate of transmission, vehicles can use network coding to improve the successful probability of transmission. $\mathrm{Su}$ et al. [2] proposed a network-coding based relay MAC protocol in vehicular networks. Ho et al. [3] use network coding to mix the packets by coding them together at every intermediate node and exploit the broadcast nature of wireless medium, so that the usefulness of each coded packet is increased. Lee et al. [4] proposed CodeTorrent, a pull-based content distribution scheme using network coding, where vehicles need to explicitly initiate requests to download a piece of content.

Three problems may occur when networking coding is adopted in vehicular networks. First, many network coding researches do not consider the error probability of data transmissions. Second, the process of network coding is to divide the data into several packets, and then for each received data, chooses a coefficient to multiply with it, and recombines these data into a code vector. If the same coefficient is chosen to multiply with different data, the destination cannot decode the original data. Third, symbol level network coding can decrease the error probability and it has a special way to decode the original data even the destination received a data with some lost symbols [5]. However, if the lost parts of each received code vector are the same, the destination cannot decode the original data.

To solve the above problems, we have proposed an overheard-based relay-assisted MAC protocol. The error probability is considered in this paper and symbol-level network coding is adopted so as to reduce the error probability. To avoid different data multiplies with the same coefficient, an overheard-based coefficient determination mechanism is proposed. If the lost parts of each received code vector are the same, the symbol acquisition process will be initiated.

The remainder of this paper is organized as follows. Preliminaries are shown in section 2. Section 3 describes the overheard-based relay-assisted MAC protocol. Section 4 presents the simulation results. Section 5 concludes this paper.

\section{PRELiminaries}

The system model is shown in section II-A and the motivation is shown in section II-B.

\section{A. System Model}

The proposed scheme are design for vehicles on a highway with several road side units (RSU) along the highway and each RSU connects with Internet. If a vehicle wants to transmit data to Internet, it has to transmit the data via an RSU. If the vehicle is out of the communication range of 


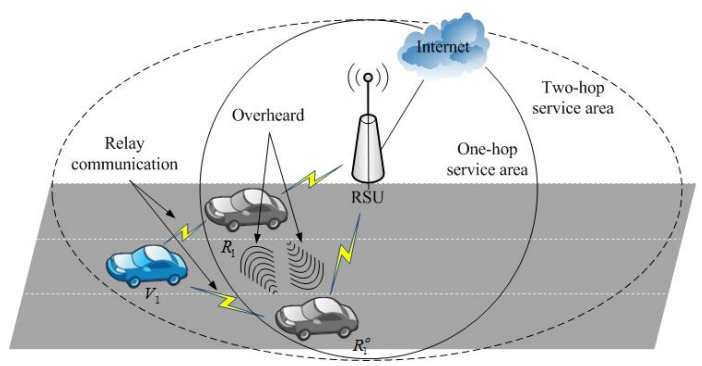

Figure 1. System architecture

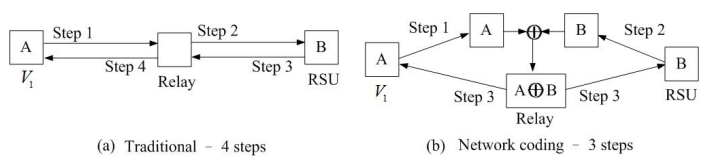

Figure 2. Traditional communications vs. network coding

an RSU, it has to find other vehicles to relay the data to the RSU. Fig. 1 shows the system model of the proposed scheme. There are a road side unit and at least three vehicles in the vehicular network. Vehicles $R_{1}$ and $R_{1}^{o}$ are in the direct communication range of the RSU, while vehicle $V_{1}$ is not in the direct communication range of the RSU and thus cannot connect to Internet via the RSU. If $V_{1}$ wants to transmit data to Internet, it needs the help of other vehicles those are in the direct communication range of the RSU. Since vehicles $R_{1}$ and $R_{1}^{o}$ are in the direct communication range of the RSU, they can act as relay vehicles and help vehicle $V_{1}$ to relay the packets to Internet via the RSU. Besides that, by the help of relay vehicles, the RSU can extend its communication range to serve more vehicles and thus less RSUs are needed to cover the same serving area. The relayed packets can be transmitted through network coding so as to decrease the amount of packets being transmitted. However, network coding has some problems which may cause decoding failure.

\section{B. Motivation}

Broadcast is a fundamental operation in communication networks. The simplest broadcast algorithm is flooding where every node broadcasts the packet when a node receives a novel packet. The implementation of flooding is simple but it may lead to higher probability of collisions and results in network congestion. Network coding is an alternative approach to resolve the problems.

In Fig. 2, vehicle $V_{1}$ wants to exchange messages with the RSU through the relay vehicle. Transmitting by traditional communications, four transmissions are needed; while transmitting by network coding, only three transmissions are needed. Hence, network coding can reduce the number of transmissions.

There are many kinds of network coding methods, like linear network coding, opportunistic network coding, symbol

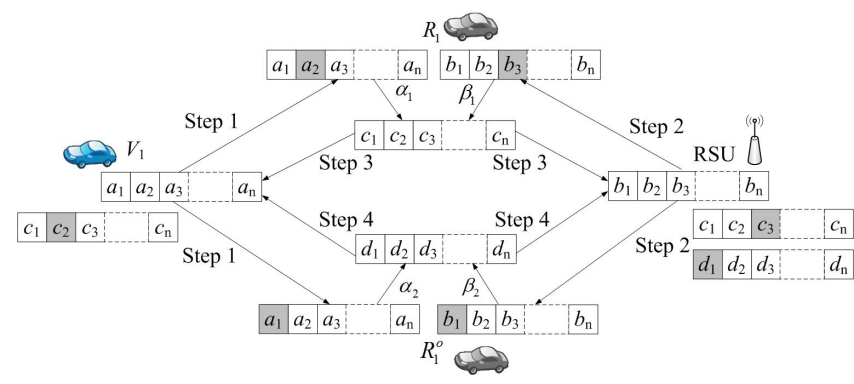

Figure 3. Symbol level network coding procedure

level network coding (SLNC), etc. The symbol level network coding can decrease the error probability and hence, it is adopted in the proposed protocol. The objective of the paper is to improve the successful probability of transmissions and reduce the number of retransmissions by using symbol level network coding.

Three problems may occur when networking coding is adopted in the vehicular networks. The first problem is that many network coding researches do not consider the error probability when transmitting data. Most of the existing works focus on how to decode the encoded packets and assume that all the transmitting packets can be successfully received. However, empirical tests show that if 10 packets have been transmitted, the destination can only receives 3.2 packets, other packets are error or lost [6]. Therefore, the error probability is considered in this paper.

Second, the process of network coding is to divide the data into several packets, and then for each code vector, chooses a coefficient to multiply with it, and recombines the multiplication results into a new code vector. After the process, the data is totally different from the original data. This process is called "encode". Through encoding, the destination needs to receive enough code vector to decode the original data by linear elimination. According to linear formula, if the coefficients are different, they will be able to decode the data. But the coefficient is randomly chosen, if the same coefficient is chosen to multiply with different code vector, the destination cannot decode the original data.

Third, symbol level network coding can decrease the error probability and it has a special way to decode the original data even the destination received a code vector with some lost symbols. If these code vectors have different lost parts, the destination still can decode these code vectors. However, if the lost parts are the same, the destination cannot decode these code vectors.

To solve the above problems, we have proposed an overheard-based relay-assisted MAC protocol. The proposed protocol can reduce delivery delay, enhance throughput and decrease the error probability of transmissions. 


\section{OVERHEARD-BASED RELAY-ASSISTED MAC Protocol Using Symbol LeVEl Network Coding}

The proposed scheme uses overheard to conquer the problems of network coding. If a vehicle wants to transmit data by the help of relay vehicles, it will choose a relay vehicle with the best transmitting quality to relay its data. Even the data can be relayed, the data may be lost because of the high error probability. A symbol level network coding procedure is adopted to solve the problem as shown in Fig. 3. Assume that vehicle $V_{1}$ is two-hop away from $R S U$. Vehicle $V_{1}$ wants to transmit data $a$ to $R S U$ and $R S U$ wants to transmit data $b$ to $V_{1}$. Data $a$ and $b$ are transmitted through relay vehicles $R_{1}$ and $R_{1}^{o}$. At first, vehicle $V_{1}$ divides data $a$ into $n$ symbols (e.g. code vector $\left.\left(a_{1}, a_{2}, \ldots, a_{n}\right)\right)$ and broadcasts these symbols to relay vehicles $R_{1}$ and $R_{1}^{o}$. Similarly, $R S U$ divides data $b$ into $n$ symbols and broadcasts these symbols to relay vehicles $R_{1}$ and $R_{1}^{o}$. After received the code vectors of $a$ and $b$, each of the relay vehicle randomly chooses two coefficients to multiply them with the code vectors of $a$ and $b$, respectively. By combining the two multiplication results, $R_{1}$ derives code vector $\left(c_{1}, c_{2}, \ldots, c_{n}\right)$, where $c_{i}=\alpha_{1} a_{i}+\beta_{1} b_{i}$ and $1 \leq i \leq n$, and $R_{1}^{o}$ derives code vector $\left(d_{1}, d_{2}, \ldots, d_{n}\right)$, where $d_{i}=\alpha_{2} a_{i}+\beta_{2} b_{i}$. Relay vehicles $R_{1}$ and $R_{1}^{o}$ then broadcast code vectors $\left(c_{1}, c_{2}, \ldots, c_{n}\right)$ and $\left(d_{1}, d_{2}, \ldots, d_{n}\right)$, respectively. After, received code vectors $\left(c_{1}, c_{2}, \ldots, c_{n}\right)$ and $\left(d_{1}, d_{2}, \ldots, d_{n}\right)$, vehicle $V_{1}$ and $R S U$ can derive the original data $b$ and $a$ by linear elimination, respectively. Even there are some lost symbols (e.g. the shaded symbols), the original data can still be derived if the coefficients and lost parts of the two code vectors are different. If the coefficients or lost parts of the two code vectors are the same, two relay vehicles are chosen to solve the problem. Relay vehicle $R_{1}$ acts as a normal relay vehicle, and relay vehicle $R_{1}^{o}$ not only relays data but also switches to promiscuous mode. By using the overheard information, relay vehicle $R_{1}^{o}$ can derive the lost parts of the data from code vectors $a, b$, and $c$ and can avoid to choose the same coefficients as vehicle $R_{1}$.

The proposed overheard-based relay-assisted MAC protocol contains three phases: The packet overheard, the overheard-based coefficient determination, and the symbol acquisition phases.

\section{A. Phase I: Packet Overheard}

In the packet overheard phase, the overheard relay vehicle $R_{1}^{o}$ will switch to promiscuous mode when vehicle $R_{1}$ is broadcasting its code vector. By using the overheard code vector, vehicle $R_{1}^{o}$ can derive the lost symbols. In Fig. 4, vehicle $R_{1}$ lost symbols $a_{1}$ and $b_{2}$; while vehicle $R_{1}^{o}$ lost symbols $a_{4}$ and $b_{3}$. With the received code vectors $\left(a_{1}, a_{2}, a_{3},{ }_{-}\right)$and $\left(b_{1}, b_{2},{ }_{-}, b_{4}\right)$, and overheard code vector $\left(c_{1},-, c_{3}, c_{4}\right)$, vehicle $R_{1}^{o}$ can derive $a_{4}$ from encoded symbol $c_{4}\left(=\alpha_{1} a_{4}+\beta_{1} b_{4}\right)$ and $b_{4}$, and derives $b_{3}$ from encoded symbol $c_{3}\left(=\alpha_{1} a_{3}+\beta_{1} b_{3}\right)$ and $a_{3}$ by linear elimination.

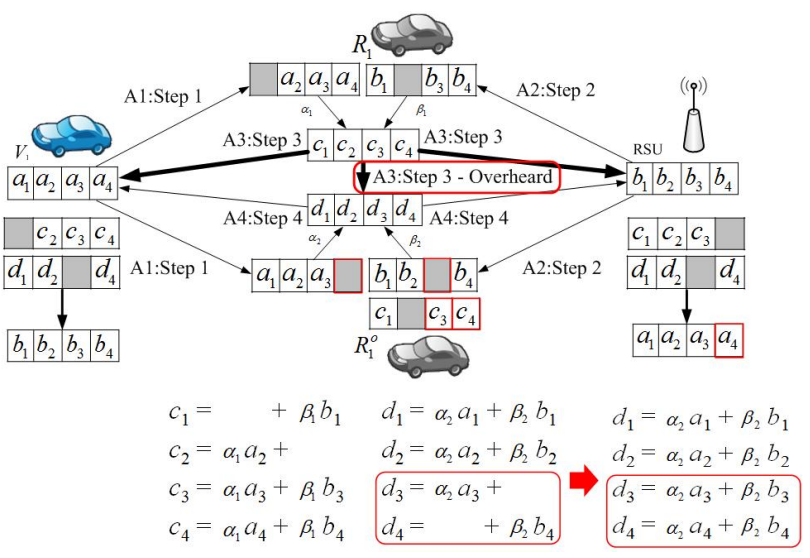

Figure 4. Packet overheard phase

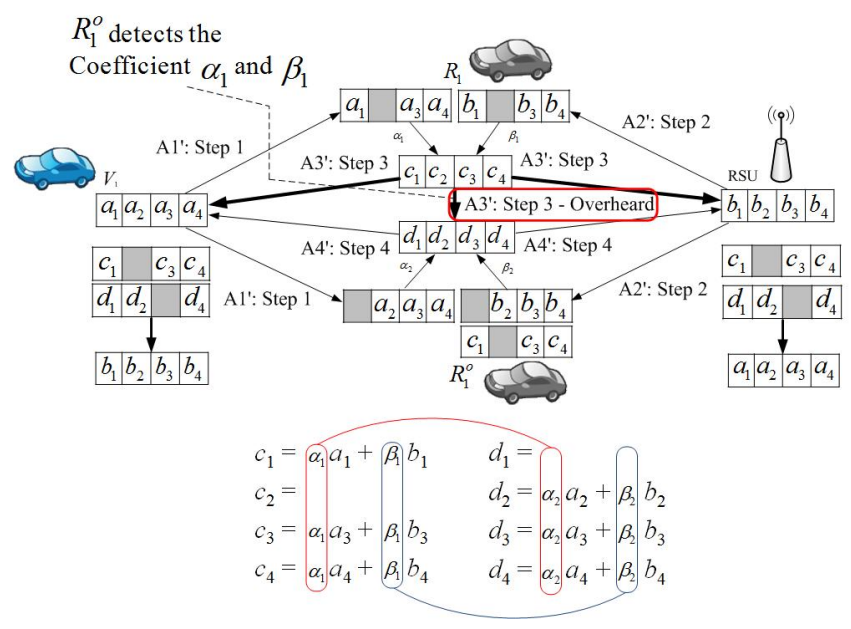

Figure 5. Overheard-based coefficient determination phase

After the derivation of $a_{4}$ and $b_{3}$, vehicle $R_{1}^{o}$ can derive code vector $\left(d_{1}, d_{2}, d_{3}, d_{4}\right)$.

\section{B. Phase II: Overheard-Based Coefficient Determination}

In the overheard-based coefficient determination phase, the overheard vehicle $R_{1}^{o}$ can use the received code vectors $\left(a_{1}, a_{2}, \ldots, a_{n}\right)$ and $\left(b_{1}, b_{2}, \ldots, b_{n}\right)$, and overheard code vector $\left(c_{1}, c_{2}, \ldots, c_{n}\right)$ to derive coefficients $\alpha_{1}$ and $\beta_{1}$ by linear elimination, and avoid choosing the same coefficients. In Fig. 5, vehicle $R_{1}^{o}$ can derive coefficients $\alpha_{1}$ and $\beta_{1}$ by solving the equations of the encoded symbols $c_{1}, c_{3}$, and $c_{4}$. Since $c_{i}=\alpha_{1} a_{i}+\beta_{1} b_{i}$ and symbols $a_{1}, a_{2}, a_{3}, b_{1}, b_{2}$, $b_{4}, c_{1}, c_{3}$, and $c_{4}$ are already known by vehicle $R_{1}^{o}$, vehicle $R_{1}^{o}$ can derive coefficients $\alpha_{1}$ and $\beta_{1}$ by linear elimination. With the value of coefficients $\alpha_{1}$ and $\beta_{1}$, vehicle $R_{1}^{o}$ can choose different coefficients $\alpha_{2}$ and $\beta_{2}$ to multiply with the code vectors $\left(a_{1}, a_{2}, a_{3}, a_{4}\right)$ and $\left(b_{1}, b_{2}, b_{3}, b_{4}\right)$ respectively, and combine the multiplication results to derive the code vector $\left(d_{1}, d_{2}, d_{3}, d_{4}\right)$. After the code vector $\left(d_{1}, d_{2}, d_{3}, d_{4}\right)$ has been derived, vehicle $R_{1}^{o}$ then broadcast the code vector 

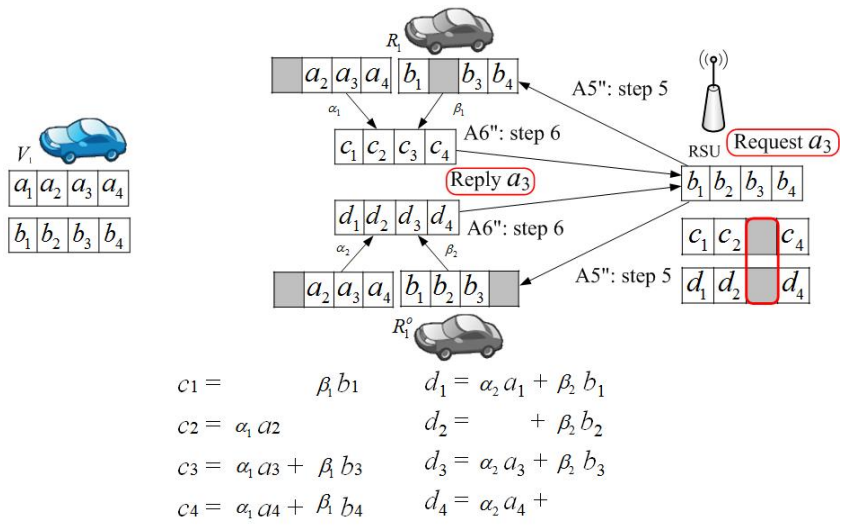

Figure 6. Symbol acquisition phase

to vehicle $V_{1}$ and $R S U$. With different coefficients, vehicle $V_{1}$ and $R S U$ will be able to derive data $b$ and $a$ by linear elimination, respectively. Without overheard relay vehicle, different coefficients cannot be guaranteed and thus vehicle $V_{1}$ and $R S U$ may not be able to recover the encoded data because linear elimination does not work with the same coefficients.

\section{Phase III: Symbol Acquisition}

In the symbol acquisition phase, if vehicle $V_{1}$ or $R S U$ has found that it cannot decode the original data (e.g. $b$ or $a$ ) because it has lost some symbols, it will send a request packet to the relay vehicles $R_{1}$ and $R_{1}^{o}$. One of the relay vehicles that has the lost symbols will reply the lost symbols. In Fig. 6, RSU has lost the encoded symbols $c_{3}$ and $d_{3}$, and thus it cannot decode the symbol $a_{3}$. Hence, it sends a request packet to the relay vehicles $R_{1}$ and $R_{1}^{o}$. Both of the relay vehicles (e.g. $R_{1}$ and $R_{1}^{o}$ ) have symbol $a_{3}$, but $R_{1}$ reply $a_{3}$ to $R S U$ earlier than $R_{1}^{o}$ does. $R_{1}^{o}$ will not send the reply. Since the packet of symbol level network coding is smaller than the packet of packet level network coding, the cost of the symbol level request is less than that of the packet level request.

\section{Simulation Results}

To evaluate the performance of the proposed protocol, we simulate the direct transmission, packet level network coding, symbol level network coding, and symbol level network coding with overheard on NCTUns 6.0 Network Simulator and Emulator with ITS module. For the ease of describing the simulation results, the direct transmission scheme is denoted as DT, the packet level network coding scheme is denoted as PLNC, the symbol level network coding scheme is denoted as SLNC, and the symbol level network coding with overheard is denoted as SLNC-O. The simulation parameters are shown in Table I.

The performance metrics observed in the simulations are shown as follows:
Table I

SIMULATION PARAMETERS

\begin{tabular}{|c|c|}
\hline Simulation scenario & Highway \\
Simulation area & $8 \mathrm{~km}$ \\
Number of a lanes & 4 \\
Transmission range & $250 \mathrm{~m}$ \\
Number of vehicles & $300 / \mathrm{h}$ \\
Beacon period & $50 \mathrm{~ms}$ \\
Vehicle velocity & $70-110 \mathrm{~km} / \mathrm{h}$ \\
\hline
\end{tabular}

- Successful probability is defined as the total number of packets that can be successfully decoded by the destination over the total number of transmitted packets.

- Average delivery delay is defined as the interval from the time the source start to transmit the packet to the time the destination successfully receive the packet.

- Throughput is defined as the amount of data being received by the destination successfully per second.

\section{A. Successful probability}

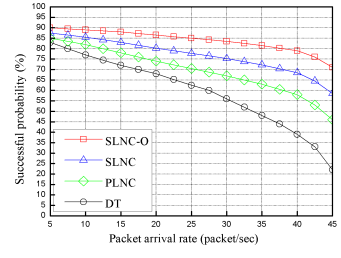

(a) Impact of packet arrival rate.

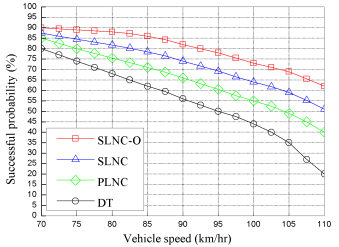

(b) Impact of vehicle speed.
Figure 7. Successful probability

Fig 7 shows the impact of packet arrival rate and vehicle speed to the successful probability. As the packet arrival rate increases, the successful probability decreases as shown in Fig 7(a), because higher packet arrival rate causes higher traffic load and more contentions and collisions may occur and thus increases the error probability and decreases the successful probability of transmissions. As the vehicle speed increases, the successful probability decreases as shown in Fig 7(b), because as the vehicle speed increases, the vehicle has less chance to connect with the RSU and the connections are more likely to be broken, and thus decreases the successful probability of transmissions. Among the four schemes, the successful probability of the SLNC-O scheme is the highest follows by the SLNC, PLNC, and DT schemes. The symbol level network coding-based schemes (e.g. SLNC-O and SLNC) performs better than the packet level network coding scheme because of their lower error probabilities of transmissions. SLNC-O scheme performs better than SLNC scheme, because there is an overheard relay vehicle in SLNC-O which can help to decode the lost symbols and thus enhance the successful probability of transmissions. All the network coding-based schemes perform better than 
DT scheme, because network coding can reduce the error probability of transmissions.

\section{B. Average delivery delay}

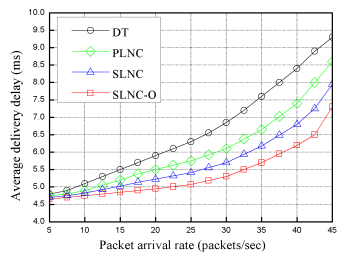

(a) Impact of packet arrival rate.

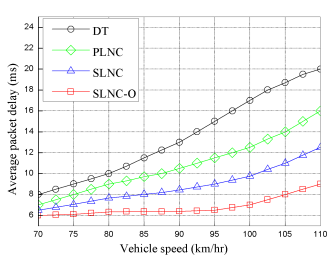

(b) Impact of vehicle speed.
Figure 8. Average delivery delay

Fig 8 shows the impact of packet arrival rate and vehicle speed to the average delivery delay. As the packet arrival rate increases, the average delivery delay also increases as shown in Fig 8(a). When a source transmits a packet, the packet could be dropped because the packet is not complete or error. Since the incomplete packet is useless, the source needs to retransmit the packet. Higher packet arrival rate causes lower successful probability of transmission and thus more retransmissions are needed. Hence, the average delivery delay of each packet is increased. As the vehicle speed increases, the average delivery delay also increases as shown in Fig 8(b), because as the vehicle speed increases, the vehicle has less chance to connect with the RSU and thus it cannot transmit and receive all the data by passing through only one RSU. Among the four schemes, the average delivery delay of the SLNC-O scheme is the lowest follows by the SLNC, PLNC, and DT schemes. The symbol level network coding-based schemes (e.g. SLNC-O and SLNC) performs better than packet level network coding scheme because of their higher successful probabilities of transmissions. SLNC-O scheme performs better than SLNC scheme, because there is an overheard relay vehicle in SLNC-O which can help to reduce the number of retransmissions. All the network coding-based schemes perform better than DT scheme, because network coding can reduce the number of transmissions.

\section{Throughput}

Fig 9 shows the impact of packet arrival rate and vehicle speed to the throughput. As the packet arrival rate increases, the throughput also increases as shown in Fig 9(a), because higher packet arrival rate causes more data to be delivered and thus more data can be received by the destination. As the vehicle speed increases, the throughput decreases as shown in Fig 8(b), because as the vehicle speed increases, more packets are lost and thus the throughput becomes lower. Among the four schemes, the throughput of the SLNC-O scheme is the highest follows by the SLNC, PLNC, and DT schemes, because lower delivery delay indicates higher

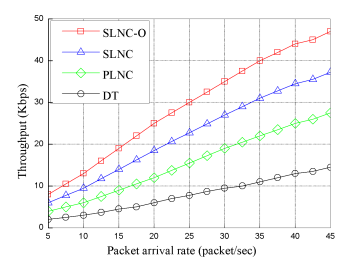

(a) Impact of packet arrival rate.

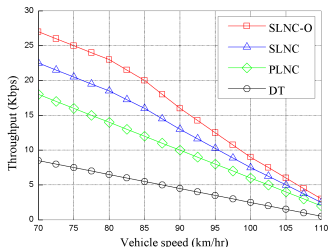

(b) Impact of vehicle speed.
Figure 9. Throughput

throughput and the average delivery delay of the SLNC-O scheme is the lowest follows by the SLNC, PLNC, and DT schemes.

\section{CONCLUSiOnS}

This paper presents an overheard-based relay-assisted MAC protocol in vehicular networks. Through symbol level network coding and the help of the overheard relay vehicle, the problems of the traditional network coding, such as error probability of transmissions, the same coefficients, and the same lost symbols, can be solved. Simulation results have shown that the proposed MAC protocol performs better than the existing network coding protocols in terms of the successful probability, average delivery delay, and throughput.

\section{ACKNOWLEDGMENT}

This research was supported by the National Science Council of the R.O.C. under grant NSC-100-2221-E-305001-MY3 and NSC-100-2221-E-305-006.

\section{REFERENCES}

[1] R. Alshwede, N. Cai, S.-Y. R. Li, and R. W. Yeung, "Network information flow: Single source," IEEE Trans. Inform. Theory, submitted for publication.

[2] H. Su and X. Zhang, "Network-coding-based relay mac protocols for drive-thru internet services in vehicular networks," in Proceedings of IEEE Global Telecommunications Conference (IEEE GLOBECOM 2010), 2010.

[3] T. Ho, M. Medard, R. Koetter, D. Karger, M. Effros, J. Shi, and B. Leong, "A random linear network coding approach to multicast," IEEE Trans. Information Theory, vol. 52, no. 10, pp. 4413-4430, Oct. 2006.

[4] U. Lee, J.-S. Park, J. Yeh, G. Pau, and M. Gerla, "Code torrent: content distribution using network coding in vanet," in Proceedings of Mobi-Share 06, 2006, pp. 1-5.

[5] S. Katti, D. Katabi, H. Balakrishnan, and M. Medard, "Symbollevel network coding for wireless mesh networks," ACM SIGCOMM Comput. Commun. Rev., vol. 38, no. 4, pp. 401-412, 2008.

[6] M. M. M. Kim, C. W. Ahn and M. Effros, "On minimizing network coding resources: An evolutionary approach," in Proceedings of NetCod, 2006. 